\title{
Efficient synthesis of polyfunctionalized carbazoles and pyrrolo[3,4-c]carbazoles via domino Diels-Alder reaction
}

\author{
Ren-Jie Fang, Chen Yan, Jing Sun*, Ying Han and Chao-Guo Yan*
}

\author{
Full Research Paper \\ Address: \\ College of Chemistry and Chemical Engineering, Yangzhou \\ University, Yangzhou 225002, China \\ Email: \\ Jing Sun* - sunjing@yu.edu.cn; Chao-Guo Yan * - cgyan@yzu.edu.cn \\ ${ }^{*}$ Corresponding author \\ Keywords: \\ carbazole; chalcone; Diels-Alder reaction; maleimide; \\ pyrrolo[3,4-c]carbazole; 3-vinylindole
}

Beilstein J. Org. Chem. 2021, 17, 2425-2432.

https://doi.org/10.3762/bjoc.17.159

Received: 27 July 2021

Accepted: 06 September 2021

Published: 16 September 2021

Associate Editor: B. Nay

(C) 2021 Fang et al.; licensee Beilstein-Institut.

License and terms: see end of document.

\begin{abstract}
The $p$-TsOH-catalyzed Diels-Alder reaction of 3-(indol-3-yl)maleimides with chalcone in toluene at $60{ }^{\circ} \mathrm{C}$ afforded two diastereoisomers of tetrahydropyrrolo[3,4-c]carbazoles, which can be dehydrogenated by DDQ oxidation in acetonitrile at room temperature to give the aromatized pyrrolo[3,4-c]carbazoles in high yields. On the other hand, the one-pot reaction of 3-(indol-3-yl)-1,3diphenylpropan-1-ones with chalcones or benzylideneacetone in acetonitrile in the presence of $p$-TsOH and DDQ resulted in polyfunctionalized carbazoles in satisfactory yields. The reaction mechanism included the DDQ oxidative dehydrogenation of 3-(indol3-yl)-1,3-diphenylpropan-1-ones to the corresponding 3-vinylindoles, their acid-catalyzed Diels-Alder reaction and sequential aromatization process.
\end{abstract}

\section{Introduction}

Carbazole is one of the most well-known privileged nitrogencontaining heterocycles. The carbazole skeleton is widely occurring in natural alkaloids and pharmacologically active compounds representing a broad spectrum of important bioactivities such as anticancer, antituberculosis, anti-protein kinase $\mathrm{C}$, antipsychotic, and antioxidative activities [1-5]. For some examples, carprofen is a nonsteroidal anti-inflammatory pharmaceutical used to treat joint pain and postoperative pain [6] (Figure 1). Ellipticine was considered to be based mainly on DNA intercalation and topoisomerase II inhibition [7]. Midostaurin and carvediol have been approved by the FDA for tumor therapy and treatment of congestive heart failure [8]. On the other hand, carbazole derivatives also have potential applications in optoelectronic materials, conducting polymers, and synthetic dyes [9-11]. Over the past decades, many efficient synthetic methodologies for functionalized carbazole derivatives have been successfully developed [12-18]. Because indoles are readily available materials, the direct extension of indoles to carbazole skeletons has a great advantage [19-27]. Therefore, the Diels-Alder reaction of activated 2-vinylindolines or 3-vinylindolines with diverse dienophiles has become the most attractive strategy for the synthesis of carbazole deriv- 
atives [28-40]. In recent years, by using the one-pot domino synthetic strategy of in situ-generated 2-vinyl- or 3-vinylindolines and sequential Diels-Alder reaction with activated dienophiles, we have successfully developed several efficient synthetic protocols for diversely functionalized tetrahydrocarbazoles and the corresponding carbazole derivatives [41-47]. To further demonstrate the synthetic application of domino Diels-Alder reactions and in continuation of our aim to providing efficient domino reactions for the synthesis of biologically important carbazole derivatives [48-53], herein we wish to report the DDQ-mediated dehydrogenative Diels-Alder reaction of 3-(indol-3-yl)maleimides and benzoyl-substituted 3-ethylindoles with readily available chalcones for the convenient synthesis of polyfunctionalized carbazole derivatives.

\section{Results and Discussion}

According to our previously established reaction conditions for the preparation of spiro[indoline-3,5'-pyrrolo[3,4-c] carbazoles] [48], an equivalent amount of 3-(indol-3-yl)maleimide with chalcone was stirred in toluene at $60{ }^{\circ} \mathrm{C}$ for two hours in the presence of $p$-toluenesulfonic acid. After workup, two diastereoisomers $\mathbf{3 a}$ and $\mathbf{3 b}$ of tetrahydropyrrolo[3,4-c]carbazoles were successfully isolated in $18 \%$ and $71 \%$ yields, respectively (Scheme 1). It should be pointed out that nearly no reaction was detected in the absence of $p$-toluenesulfonic acid. The structures of both products were fully characterized by various spectroscopy methods and confirmed by determination of their single crystal structures (Figure 2 and Figure 3).

From Figure 2, it can be seen that the phenyl group and the adjacent $p$-chlorobenzoyl group are trans-oriented. Additionally, the phenyl group is cis-oriented to the 1-benzylpyrrolidine2,5-dione ring in compound 3a. On the other hand, for compound 3b (Figure 3), it can be seen that the phenyl group is trans-oriented to both, the $p$-chlorobenzoyl group and the 1-benzylpyrrolidine-2,5-dione ring in this compound. Thus, the isomers $\mathbf{3 a}$ and $\mathbf{3 b}$ are diastereoisomers. It is known that the starting chalcones usually have $E$-configuration. The phenyl group and $p$-chlorobenzoyl group still exist in the trans-position in both diastereoisomers $\mathbf{3 a}$ and $\mathbf{3 b}$ as in the starting chalcone. This result clearly showed that this acid-catalyzed cycloaddition reaction proceeded through a concerted Diels-Alder reaction mechanism.

The acid-catalyzed Diels-Alder reaction afforded a mixture of two diastereoisomers, which decreased the synthetic value of the reaction. Thus, after the first step reaction, a DDQ dehydrogenation reaction was carried out in acetonitrile at room temper-<smiles>C[C@H](C(=O)O)c1ccc2c(c1)[nH]c1ccc(Cl)cc12</smiles>

carpofen<smiles>Cc1c2cnccc2c(C)c2c1[nH]c1ccccc12</smiles>

ellipticine<smiles>COc1ccccc1OCCNC[C@H](O)COc1cccc2[nH]c3ccccc3c12</smiles>

carvediol<smiles></smiles>

midostaurin

Figure 1: Representative bioactive carbazole derivatives.<smiles>[X]n1cc(C2=CC(=O)N(Cc3ccccc3)C2=O)c2ccccc21</smiles>

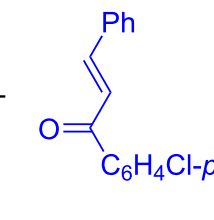

2<smiles>Cn1c2c(c3ccccc31)C1C(=O)N(Cc3ccccc3)C(=O)C1C(c1ccccc1)C2C(=O)CCCCO</smiles>

3a $(18 \%)$<smiles>Cn1c2c(c3ccccc31)C1C(=O)N(Cc3ccccc3)C(=O)C1[C@@H](c1ccccc1)C2C(=O)c1ccccc1</smiles>

3b (71\%) 


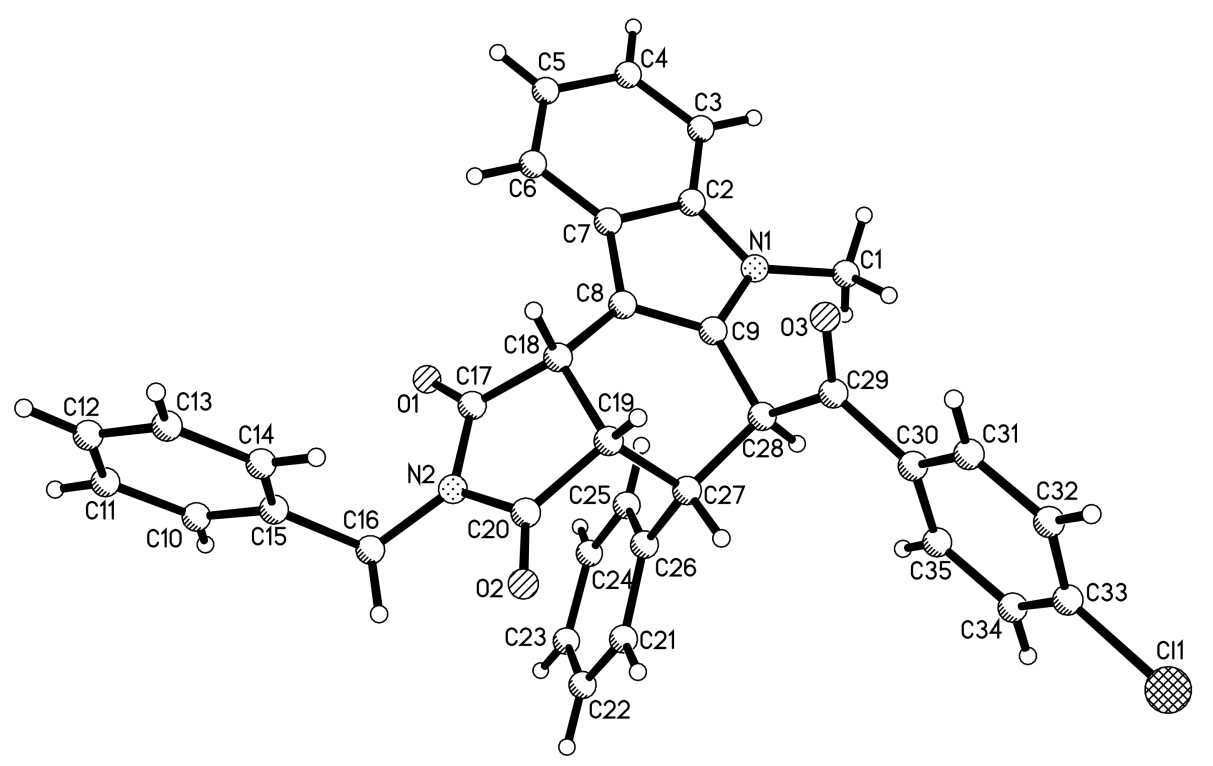

Figure 2: Single crystal structure of the isomer $\mathbf{3 a}$.

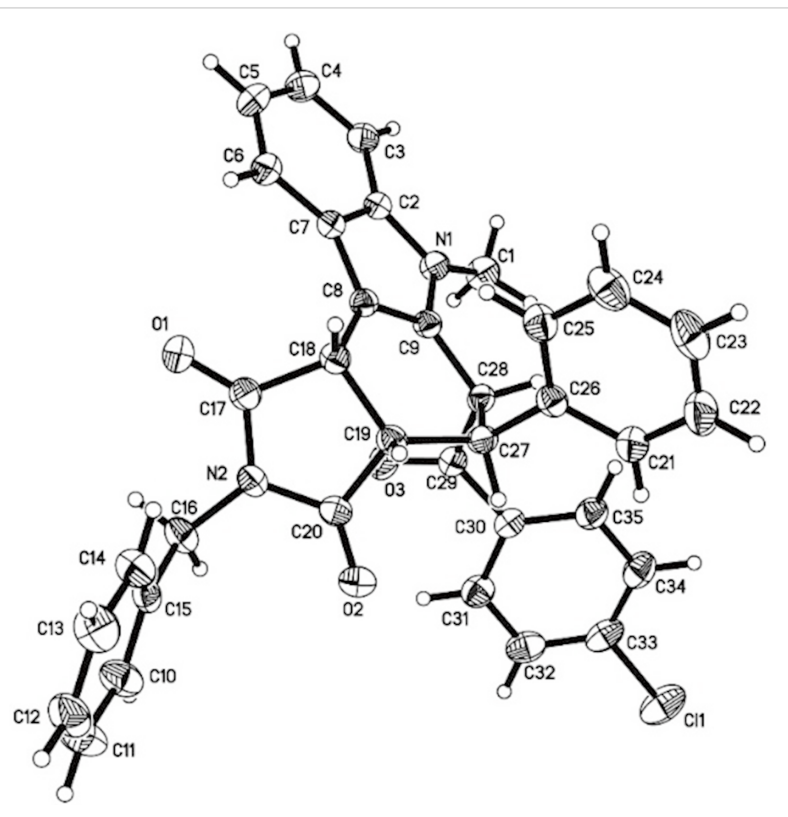

Figure 3: Single crystal structure of the isomer $\mathbf{3 b}$.

ature. A series of aromatized pyrrolo[3,4-c]carbazoles $4 \mathbf{a}-\mathbf{l}$ were successfully synthesized by the one-pot two-step reaction and the results are summarized in Table 1. All reactions proceeded smoothly to give the corresponding pyrrolo[3,4$c$ carbazoles 4a-l in satisfactory yields. Indole itself and $\mathrm{N}$-methylindole could also be successfully employed in the reaction. The $\mathrm{N}-\mathrm{Me}, \mathrm{N}-\mathrm{Ph}$, and $\mathrm{N}-\mathrm{Bn}$ substitution in the maleimide moiety showed only a marginal effect on the reaction outcome. Various chalcones with electron-donating methyl and methoxy groups and electron-withdrawing $m$-chloro and $p$-chloro substituents gave the products in good yields. However, the nitro-substituted chalcone gave the product $\mathbf{4 h}$ in a slightly lower yield. The structures of the pyrrolo[3,4$c$ carbazoles $\mathbf{4 a - 1}$ were established by various spectroscopy methods. Further, the single crystal structure of compound $\mathbf{4} \mathbf{g}$ was determined by X-ray diffraction (Figure 4). It can be seen that the ring of pyrrolo[3,4-c]carbazole exists in a slightly twisted plane. The dihedral angles of the phenyl and the benzoyl group to the central benzene ring are $72.018^{\circ}$ and $88.402^{\circ}$.

To further expand the scope of this domino Diels-Alder reaction, another kind of 3-vinylindoles was employed in the onepot reaction. First, the 3-(indol-3-yl)-1,3-diphenylpropan-1-ones prepared through Friedel-Crafts alkylation of indole with chalcones, were oxidized by DDQ in acetonitrile to generate in situ the expected active diene, indole-substituted chalcones. Then, the $p$-TsOH-catalyzed Diels-Alder reaction of indole-chalcones with second chalcones and sequential aromatization through DDQ dehydrogenation resulted in the polyfunctionalized carbazoles 6a-1 in good yields (Table 2). Additionally, the similar reaction with benzylideneacetone gave the desired carbazoles $\mathbf{6 m}$ and $\mathbf{6 n}$ albeit in significantly lower yields. Thus, this one-pot domino reaction successfully constructed carbazoles with four substituents on the benzene ring from the corresponding indole derivatives with two molecules of chalcones. It should be pointed out that the Diels-Alder reaction resulted in a complex mixture comprising four diastereoisomers of the tetrahydrocarbazoles, which was very difficult to 
Table 1: Synthesis of pyrrolo[3,4-c]carbazoles 4a-I. ${ }^{a}$<smiles>[R1]N1C(=O)C=C(c2ccn([R1])c2)C1=O</smiles><smiles>[Y17]C(=O)/C=C/[Al]</smiles><smiles>[Y10]C(=O)c1c2c(c3c4ccccc4n([R])c3c1[Y19])C(=O)N([R])C2=O</smiles>

$4 a-1$

\begin{tabular}{lllllll}
\hline entry & compound & $\mathrm{R}^{1}$ & $\mathrm{R}^{2}$ & $\mathrm{Ar}^{1}$ & $\mathrm{Ar}^{2}$ & yield (\%) \\
\hline 1 & $\mathbf{4 a}$ & $\mathrm{H}$ & $\mathrm{Ph}$ & $p-\mathrm{CH}_{3} \mathrm{C}_{6} \mathrm{H}_{4}$ & $\mathrm{C}_{6} \mathrm{H}_{5}$ & 87 \\
2 & $\mathbf{4 b}$ & $\mathrm{CH}_{3}$ & $\mathrm{CH}_{3}$ & $p-\mathrm{ClC}_{6} \mathrm{H}_{4}$ & $\mathrm{C}_{6} \mathrm{H}_{5}$ & 89 \\
3 & $\mathbf{4}$ & $\mathrm{CH}_{3}$ & $\mathrm{CH}_{3}$ & $\mathrm{C}_{6} \mathrm{H}_{5}$ & $p-\mathrm{CH}_{3} \mathrm{C}_{6} \mathrm{H}_{5}$ & 92 \\
4 & $\mathbf{4 d}$ & $\mathrm{CH}_{3}$ & $\mathrm{CH}_{3}$ & $p-\mathrm{CH}_{3} \mathrm{C}_{6} \mathrm{H}_{4}$ & $p-\mathrm{CH}_{3} \mathrm{C}_{6} \mathrm{H}_{4}$ & 91 \\
5 & $\mathbf{4 e}$ & $\mathrm{CH}_{3}$ & $\mathrm{Ph}$ & $\mathrm{C}_{6} \mathrm{H}_{5}$ & $\mathrm{CH}_{3}$ & 85 \\
6 & $\mathbf{4}$ & $\mathrm{CH}_{3}$ & $\mathrm{Ph}$ & $\mathrm{C}_{6} \mathrm{H}_{5}$ & $\mathrm{C}_{6} \mathrm{H}_{5}$ & 84 \\
7 & $\mathbf{4 g}$ & $\mathrm{CH}_{3}$ & $\mathrm{Ph}$ & $p-\mathrm{CH}_{3} \mathrm{C}_{6} \mathrm{H}_{4}$ & $\mathrm{C}_{6} \mathrm{H}_{5}$ & 93 \\
8 & $\mathbf{4}$ & $\mathrm{CH}$ & $\mathrm{Ch}$ & $m-\mathrm{NO}_{2} \mathrm{C}_{6} \mathrm{H}_{4}$ & $p-\mathrm{CH}_{3} \mathrm{C}_{6} \mathrm{H}_{4}$ & 76 \\
9 & $\mathbf{4 i}$ & $\mathrm{CH}_{3}$ & $\mathrm{Ph}$ & $p-\mathrm{CH}_{3} \mathrm{C}_{6} \mathrm{H}_{4}$ & $p-\mathrm{CH}_{3} \mathrm{OC}_{6} \mathrm{H}_{4}$ & 86 \\
10 & $\mathbf{4 j}$ & $\mathrm{CH}_{3}$ & $\mathrm{Ph}$ & $p-\mathrm{CH}_{3} \mathrm{C}_{6} \mathrm{H}_{4}$ & $m-\mathrm{ClC}_{6} \mathrm{H}_{4}$ & 84 \\
11 & $\mathbf{4 k}$ & $\mathrm{CH}_{3}$ & $\mathrm{Bn}$ & $p-\mathrm{CH}_{3} \mathrm{C}_{6} \mathrm{H}_{4}$ & $0 \mathrm{C}_{6} \mathrm{H}_{5}$ & 93 \\
12 & $\mathbf{4}$ & $\mathrm{CH}_{3}$ & $\mathrm{Bn}$ & $\mathrm{C}_{6} \mathrm{H}_{5}$ & $p-\mathrm{ClC}_{6} \mathrm{H}_{4}$ & 87 \\
\hline
\end{tabular}

aReaction conditions: 1) 3-(indol-3-yl)maleimide (1.0 mmol), chalcone (1.0 mmol), toluene $(10.0 \mathrm{~mL}), p$ - TsOH $\left.(0.2 \mathrm{mmol}), 80{ }^{\circ} \mathrm{C}, 2 \mathrm{~h} ; 2\right) \mathrm{DDQ}$ $(1.2 \mathrm{mmol}), \mathrm{CH}_{3} \mathrm{CN}(10.0 \mathrm{~mL}), \mathrm{rt}, 2 \mathrm{~h}$. blsolated yields.

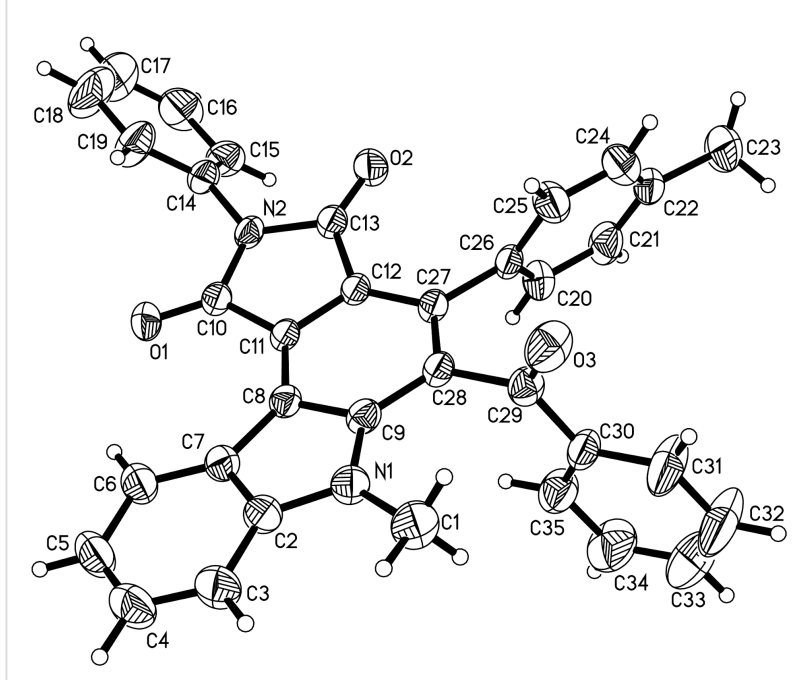

Figure 4: Single crystal structure of the isomer $\mathbf{4 g}$.

separate. After oxidation with DDQ, the aromatized carbazole derivatives $\mathbf{6 a}-\mathbf{n}$ were easily obtained as single products in good yields. The chemical structures of the carbazoles were fully characterized by ${ }^{1} \mathrm{H}$ NMR, ${ }^{13} \mathrm{C}$ NMR, IR, and HRMS spectra.
To explain the formation of the products, a plausible reaction mechanism was proposed in Scheme 2 on the basis of the previously reported reaction $[48,53]$. Firstly, the DDQ oxidative dehydrogenation of 3-(indol-3-yl)-1,3-diphenylpropan-1-one gave the expected indole-substituted chalcone $\mathbf{A}$, which comprises the desired 3-vinylindole scaffold as the reactive diene. In the meantime, the carbonyl group of the chalcone is protonated to give the activated dienophile in the presence of $p$-toluenesulfonic acid. Secondly, the Diels-Alder reaction of indolechalcone $\mathbf{A}$ with the dienophile results in the tetrahydrocarbazole $\mathbf{B}$ having an exocyclic $\mathrm{C}=\mathrm{C}$ bond. Thirdly, a new tetrahydrocarbazole intermediate $\mathbf{C}$ is formed by a $1,3-\mathrm{H}$ shifting process. The resulting tetrahydrocarbazole intermediate (C) might be a mixture of several possible diastereoisomers because it has four substituents on the cyclohexenyl ring. After a further DDQ oxidation, the aromatized carbazole $\mathbf{6}$ is successfully produced as the final product.

\section{Conclusion}

In summary, we have investigated the domino Diels-Alder reaction of 3-(indol-3-yl)maleimides and in situ-generated indole-chalcones with dienophilic chalcones. This one-pot twostep reaction successfully provided the polyfunctionalized carbazole derivatives in an extremely simple and highly effi- 
Table 2: Synthesis of the polysubstituted carbazoles $6 \mathbf{a}-\mathbf{n} .^{\text {a }}$<smiles>CCCCCCCNC(=O)CC([Al])c1cn(C)c2ccccc12</smiles>

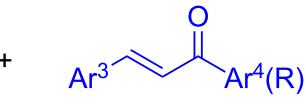

1) $\mathrm{DDQ}, \mathrm{MeCN}, 40^{\circ} \mathrm{C}, 0.5 \mathrm{~h}$ 2) $p$ - TsOH, MeCN, $90^{\circ} \mathrm{C}, 24 \mathrm{~h}$ 3) $D D Q, M e C N, r t, 1 \mathrm{~h}$

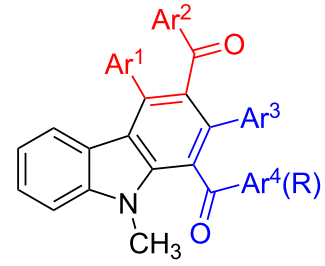

6a-n

\begin{tabular}{lllllll}
\hline entry & compound & $\mathrm{Ar}^{1}$ & $\mathrm{Ar}^{2}$ & $\mathrm{Ar}^{3}$ & $\mathrm{Ar}^{4}(\mathrm{R})$ & yield (\%) \\
\hline 1 & $\mathbf{6}$ & $\mathrm{C}_{6} \mathrm{H}_{5}$ & $\mathrm{C}_{6} \mathrm{H}_{5}$ & $\mathrm{C}_{6} \mathrm{H}_{5}$ & $\mathrm{C}_{6} \mathrm{H}_{5}$ & 61 \\
2 & $\mathbf{6 b}$ & $p-\mathrm{CH}_{3} \mathrm{C}_{6} \mathrm{H}_{4}$ & $p-\mathrm{CH}_{3} \mathrm{C}_{6} \mathrm{H}_{4}$ & $p-\mathrm{CH}_{3} \mathrm{C}_{6} \mathrm{H}_{4}$ & $p-\mathrm{CH}_{3} \mathrm{C}_{6} \mathrm{H}_{4}$ & 63 \\
3 & $\mathbf{6 c}$ & $p-\mathrm{CH}_{3} \mathrm{C}_{6} \mathrm{H}_{4}$ & $p-\mathrm{CH}_{3} \mathrm{C}_{6} \mathrm{H}_{4}$ & $p-\mathrm{ClC}_{6} \mathrm{H}_{4}$ & $p-\mathrm{CH}_{3} \mathrm{C}_{6} \mathrm{H}_{4}$ & 68 \\
4 & $\mathbf{6 d}$ & $p-\mathrm{CH}_{3} \mathrm{C}_{6} \mathrm{H}_{4}$ & $p-\mathrm{CH}_{3} \mathrm{C}_{6} \mathrm{H}_{4}$ & $o-\mathrm{CH}_{3} \mathrm{C}_{6} \mathrm{H}_{4}$ & $p-\mathrm{CH}_{3} \mathrm{OC}_{6} \mathrm{H}_{4}$ & 64 \\
5 & $\mathbf{6 e}$ & $p-\mathrm{CH}_{3} \mathrm{OC}_{6} \mathrm{H}_{4}$ & $p-\mathrm{CH}_{3} \mathrm{C}_{6} \mathrm{H}_{4}$ & $o-\mathrm{CH}_{3} \mathrm{C}_{6} \mathrm{H}_{4}$ & $p-\mathrm{CH}_{3} \mathrm{OC}_{6} \mathrm{H}_{4}$ & 62 \\
6 & $\mathbf{6}$ & $p-\mathrm{CH}_{3} \mathrm{OC}_{6} \mathrm{H}_{4}$ & $p-\mathrm{CH}_{3} \mathrm{C}_{6} \mathrm{H}_{4}$ & $p-\mathrm{CH}_{3} \mathrm{OC}_{6} \mathrm{H}_{4}$ & $p-\mathrm{ClC}_{6} \mathrm{H}_{4}$ & 66 \\
7 & $\mathbf{6 g}$ & $p-\mathrm{ClC}_{6} \mathrm{H}_{4}$ & $p-\mathrm{CH}_{3} \mathrm{C}_{6} \mathrm{H}_{4}$ & $\mathrm{C}_{6} \mathrm{H}_{5}$ & $\mathrm{C}_{6} \mathrm{H}_{5}$ & 59 \\
8 & $\mathbf{6}$ & $p-\mathrm{ClC}_{6} \mathrm{H}_{4}$ & $p-\mathrm{CH}_{3} \mathrm{C}_{6} \mathrm{H}_{4}$ & $p-\mathrm{ClC}_{6} \mathrm{H}_{4}$ & $p-\mathrm{CH}_{3} \mathrm{C}_{6} \mathrm{H}_{4}$ & 60 \\
9 & $\mathbf{6 i}$ & $p-\mathrm{ClC}_{6} \mathrm{H}_{4}$ & $p-\mathrm{CH}_{3} \mathrm{C}_{6} \mathrm{H}_{4}$ & $p-\mathrm{NO}_{2} \mathrm{C}_{6} \mathrm{H}_{4}$ & $p-\mathrm{CH}_{3} \mathrm{OC}_{6} \mathrm{H}_{4}$ & 69 \\
10 & $\mathbf{6 j}$ & $o-\mathrm{ClC}_{6} \mathrm{H}_{4}$ & $p-\mathrm{CH}_{3} \mathrm{C}_{6} \mathrm{H}_{4}$ & $p-\mathrm{CH}_{3} \mathrm{OC}_{6} \mathrm{H}_{4}$ & $p-\mathrm{ClC}_{6} \mathrm{H}_{4}$ & 63 \\
11 & $\mathbf{6}$ & $p-\mathrm{CH}_{3} \mathrm{OC}_{6} \mathrm{H}_{4}$ & $p-\mathrm{ClC}_{6} \mathrm{H}_{4}$ & $m-\mathrm{ClC}_{6} \mathrm{H}_{4}$ & $p-\mathrm{CH}_{3} \mathrm{C}_{6} \mathrm{H}_{4}$ & 61 \\
12 & $\mathbf{6 l}$ & $p-\mathrm{CH}_{3} \mathrm{OC}_{6} \mathrm{H}_{4}$ & $p-\mathrm{ClC}_{6} \mathrm{H}_{4}$ & $p-\mathrm{CH}_{3} \mathrm{OC}_{6} \mathrm{H}_{4}$ & $p-\mathrm{ClC}_{6} \mathrm{H}_{4}$ & 62 \\
13 & $\mathbf{6 m}$ & $\mathrm{C}_{6} \mathrm{H}_{5}$ & $\mathrm{C}_{6} \mathrm{H}_{5}$ & $\mathrm{C}_{6} \mathrm{H}_{5}$ & $c \mathrm{CH}_{3}$ & 31 \\
14 & $\mathbf{6 n}$ & $p-\mathrm{ClC}_{6} \mathrm{H}_{4}$ & $p-\mathrm{CH}_{3} \mathrm{C}_{6} \mathrm{H}_{4}$ & $\mathrm{C}_{6} \mathrm{H}_{5}$ & $c \mathrm{CH}_{3}$ & 32 \\
\hline
\end{tabular}

aReaction conditions: 1) 3-(indol-3-yl)-1,3-diphenylpropan-1-one (0.6 mmol), chalcone (0.5 mmol), DDQ (0.72 mmol), MeCN (15.0 mL), rt, $0.5 \mathrm{~h}$; 2) $p$ - TsOH (0.06 mmol), reflux, $4 \mathrm{~h}$; 3) DDQ (0.6 mmol), rt, $1 \mathrm{~h}$. blsolated yields.<smiles>Cn1cc(C([Al])CC(=O)[AlH2])c2ccccc21</smiles>

\section{$\stackrel{\mathrm{DDQ}}{\longrightarrow}$}<smiles>CCCCC(=O)/C=C(\[Al])c1cn(C)c2ccccc12</smiles>

A

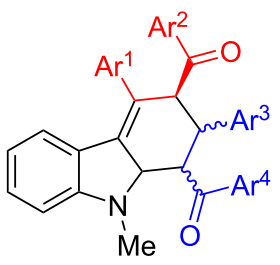

B

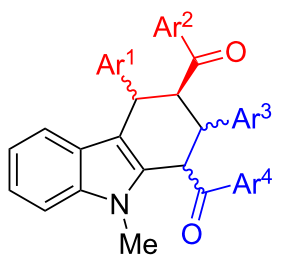

C

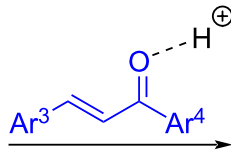

$[1,3] \mathrm{H}$ shift

\section{DDQ}<smiles>[Y19]C(=O)c1c([AlH2])c(C(=O)[AlH2])c2c(c1[Y17])c1ccccc1n2C</smiles>

6

Scheme 2: Proposed domino reaction mechanism for the formation of carbazoles 6.

cient fashion. This protocol has the advantages of using readily available starting reagents, simple manufacture, high efficiency and atomic economy. The unusual feature of this reaction is the normal electron-demand Diels-Alder reaction between elec- tron-deficient dienes such as (3-(indol-3-yl)maleimides and indole-chalcone to electron-deficient dienophilic chalcones. The potential applications of this reaction in organic and medicinal chemistry might be significant. 


\section{Experimental}

1. General procedure for the preparation of the carbazoles 4a-l: To a round-bottomed flask was added 3-(indol-3-yl)maleimide (1.0 mmol), chalcone (1.0 mmol), $p$-toluenesulfonic acid $(0.2 \mathrm{mmol})$, and toluene $(10.0 \mathrm{~mL})$. The solution was heated to $60{ }^{\circ} \mathrm{C}$ for two hours. After removing the solvent by rotatory evaporation, DDQ $(1.2 \mathrm{mmol})$ and acetonitrile $(10.0 \mathrm{~mL})$ were added and the mixture was stirred at room temperature for two hours. After removing the solvent, the residue was subjected to column chromatography with petroleum ether and ethyl acetate $8: 1(\mathrm{v} / \mathrm{v})$ as eluent to give the pure products for analysis.

5-Benzoyl-2-phenyl-4-(p-tolyl)pyrrolo[3,4-c]carbazole1,3(2H,6H)-dione (4a): green solid, $440 \mathrm{mg}, 87 \%$; mp 234-236 ${ }^{\circ} \mathrm{C}$; ${ }^{1} \mathrm{H}$ NMR (400 MHz, $\left.\mathrm{CDCl}_{3}\right) \delta 9.24$ (s, $\left.1 \mathrm{H}, \mathrm{ArH}\right)$, $9.15(\mathrm{~d}, J=8.0 \mathrm{~Hz}, 1 \mathrm{H}, \operatorname{ArH}), 7.60-7.56(\mathrm{~m}, 1 \mathrm{H}, \mathrm{ArH})$, 7.53-7.44 (m, 7H, ArH), 7.42-7.31 (m, 3H, ArH), 7.21-7.14 (m, 4H, ArH), 6.92 (d, J = 7.6 Hz, 2H, $\mathrm{ArH}), 2.18$ (s, 3H, $\mathrm{CH}_{3}$ ); ${ }^{13} \mathrm{C}\left\{{ }^{1} \mathrm{H}\right\}$ NMR $\left(100 \mathrm{MHz}, \mathrm{CDCl}_{3}\right) \delta 198.0,167.3,142.9$, $141.7,138.2$, 137.6, 137.2, 132.9, 131.9, 131.7, 130.7, 129.1, 129.0, 128.8, 128.2, 127.9, 127.8, 127.7, 126.7, 126.1, 125.6, 121.7, 120.7, 120.3, 119.2, 111.3, 21.1; IR (KBr) v: 2988, 1786, 1734, 1611, 1485, 1456, 1357, 1314, 1185, 1021, 988, 786, $734 \mathrm{~cm}^{-1}$; HRMS-ESI-TOF $(\mathrm{m} / \mathrm{z}):[\mathrm{M}+\mathrm{Na}]^{+}$calcd for $\mathrm{C}_{34} \mathrm{H}_{22} \mathrm{NaN}_{2} \mathrm{O}_{3}$, 529.1523; found, 529.1512.

2. General procedure for the preparation of carbazoles 6a-n: To a round-bottomed flask were added 3-(indol-3-yl)1,3-diphenylpropan-1-one (0.6 mmol), DDQ ( $0.72 \mathrm{mmol})$, and acetonitrile $(15.0 \mathrm{~mL})$. The mixture was stirred at room temperature for $30 \mathrm{~min}$. Then, the chalcone $(0.5 \mathrm{mmol})$ and $p$-toluenesulfonic acid $(0.06 \mathrm{mmol})$ were added and the solution was refluxed for four hours. After cooling to room temperature, DDQ $(0.6 \mathrm{mmol})$ was added and the mixture was stirred at room temperature for one hour. After removing the solvent by rotatory evaporation at reduced pressure, the residue was subjected to column chromatography with a mixture of petroleum ether, ethyl acetate and methylene dichloride 20:1:5 (v/v/v) to give the pure products for analysis.

(9-Methyl-2,4-diphenyl-9H-carbazole-1,3-diyl)bis(phenylmethanone) (6a): White solid, $165 \mathrm{mg}, 61 \%$; mp 249-251 ${ }^{\circ} \mathrm{C}$; ${ }^{1} \mathrm{H} \mathrm{NMR}\left(400 \mathrm{MHz}, \mathrm{CDCl}_{3}\right) \delta 7.64(\mathrm{~d}, J=7.6 \mathrm{~Hz}, 3 \mathrm{H}, \mathrm{ArH})$, $7.43(\mathrm{t}, J=7.8 \mathrm{~Hz}, 2 \mathrm{H}, \mathrm{ArH}), 7.39-7.34$ (m, 6H, ArH), $7.28-7.24$ (m, 3H, ArH), 7.09 (t, $J=7.6 \mathrm{~Hz}, 2 \mathrm{H}, \mathrm{ArH}), 7.01$ (s, $2 \mathrm{H}, \operatorname{ArH}), 6.96(\mathrm{t}, J=7.8 \mathrm{~Hz}, 2 \mathrm{H}, \operatorname{ArH}), 6.84(\mathrm{~d}, J=6.8 \mathrm{~Hz}$, $4 \mathrm{H}, \mathrm{ArH}), 3.64\left(\mathrm{~s}, 3 \mathrm{H}, \mathrm{CH}_{3}\right) ;{ }^{13} \mathrm{C} \mathrm{NMR}\left(400 \mathrm{MHz}, \mathrm{CDCl}_{3}\right) \delta$ 198.5, 198.4, 142.4, 138.9, 138.7, 137.6, 137.5, 136.8, 135.9, $135.2,133.3,132.2,131.5,131.4,129.5,129.2,128.3,128.2$, $127.8,127.6,127.0,126.9,126.4,122.4,122.0,121.8,121.6$, 119.6, 108.7, 32.1; IR (KBr) v: 3057, 3023, 2907, 2360, 2339,
1720, 1605, 1482, 1320, 1267, 1172, 1009, 936, 805, 743, 612, $447 \mathrm{~cm}^{-1}$; HRMS-ESI $(\mathrm{m} / \mathrm{z}):[\mathrm{M}+\mathrm{Na}]^{+}$calcd for $\mathrm{C}_{39} \mathrm{H}_{27} \mathrm{NO}_{2}$, 564.1934; found, 564.1926.

The crystallographic data of the compounds 3a (CCDC 2099074), 3b (CCDC 2099075), and 4g (CCDC 2099076) have been deposited at the Cambridge Crystallographic Database Centre (http://www.ccdc.cam.ac.uk).

\section{Supporting Information}

\section{Supporting Information File 1}

Characterization data and ${ }^{1} \mathrm{H}$ NMR, ${ }^{13} \mathrm{C}$ NMR, and HRMS spectra of the synthesized compounds.

[https://www.beilstein-journals.org/bjoc/content/ supplementary/1860-5397-17-159-S1.pdf]

\section{Funding}

This work was financially supported by the National Natural Science Foundation of China (Nos. 21572196, 21871227) and the Priority Academic Program Development of Jiangsu Higher Education Institutions (No. BK2013016).

\section{ORCID ${ }^{\circledR} \mathrm{iDs}$}

Chao-Guo Yan - https://orcid.org/0000-0002-2777-9582

\section{Preprint}

A non-peer-reviewed version of this article has been previously published as a preprint: https://doi.org/10.3762/bxiv.2021.53.v1

\section{References}

1. Thevissen, K.; Marchand, A.; Chaltin, P.; Meert, E. M. K.; Cammue, B. P. A. Curr. Med. Chem. 2009, 16, 2205-2211. doi:10.2174/092986709788612701

2. Dhara, K.; Mandal, T.; Das, J.; Dash, J. Angew. Chem., Int. Ed. 2015, 54, 15831-15835. doi:10.1002/anie.201508746

3. He, W.; Wang, P.; Chen, J.; Xie, W. Org. Biomol. Chem. 2020, 18, 1046-1056. doi:10.1039/c9ob02627d

4. Hesse, R.; Kataeva, O.; Schmidt, A. W.; Knölker, H.-J. Chem. - Eur. J. 2014, 20, 9504-9509. doi:10.1002/chem.201403645

5. Shaikh, M.; Karpoormath, R.; Thapliyal, N.; Rane, R.; Palkar, M.; Faya, A. M.; Patel, H.; Alwan, W.; Jain, K.; Hampannavar, G. Anti-Cancer Agents Med. Chem. 2015, 15, 1049-1065. doi:10.2174/1871520615666150113105405

6. Favia, A. D.; Habrant, D.; Scarpelli, R.; Migliore, M.; Albani, C.; Bertozzi, S. M.; Dionisi, M.; Tarozzo, G.; Piomelli, D.; Cavalli, A.; De Vivo, M. J. Med. Chem. 2012, 55, 8807-8826. doi:10.1021/jm3011146

7. Deng, C.; Liu, Y.; Xu, M.; Xie, K.; Liu, S. Org. Biomol. Chem. 2021, 19, 1395-1403. doi:10.1039/d0ob02527e 
8. Caruso, A.; Sinicropi, M. S.; Lancelot, J.-C.; El-Kashef, H.; Saturnino, C.; Aubert, G.; Ballandonne, C.; Lesnard, A.; Cresteil, T.; Dallemagne, P.; Rault, S. Bioorg. Med. Chem. Lett. 2014, 24, 467-472. doi:10.1016/j.bmcl.2013.12.047

9. Wang, C.; Dong, H.; Hu, W.; Liu, Y.; Zhu, D. Chem. Rev. 2012, 112, 2208-2267. doi:10.1021/cr100380z

10. Bryden, M. A.; Zysman-Colman, E. Chem. Soc. Rev. 2021, 50 , 7587-7680. doi:10.1039/d1cs00198a

11. Cha, M. S.; Park, J. E.; Kim, S.; Han, S.-H.; Shin, S.-H.; Yang, S. H.; Kim, T.-H.; Yu, D. M.; So, S.; Hong, Y. T.; Yoon, S. J.; Oh, S.-G.; Kang, S. Y.; Kim, O.-H.; Park, H. S.; Bae, B.; Sung, Y.-E.; Cho, Y.-H.; Lee, J. Y. Energy Environ. Sci. 2020, 13, 3633-3645. doi:10.1039/d0ee01842b

12. Knölker, H.-J.; Reddy, K. R. Chem. Rev. 2002, 102, 4303-4428. doi:10.1021/cr020059j

13. Schmidt, A. W.; Reddy, K. R.; Knölker, H.-J. Chem. Rev. 2012, 112, 3193-3328. doi:10.1021/cr200447s

14. Lancianesi, S.; Palmieri, A.; Petrini, M. Chem. Rev. 2014, 114, 7108-7149. doi:10.1021/cr400676v

15. Liu, D.; Huang, J.; Fu, Z.; Huang, W. Green Chem. 2019, 21, 968-972. doi:10.1039/c9gc00064j

16. Faltracco, M.; Ortega-Rosales, S.; Janssen, E.; Cioc, R. C.; Vande Velde, C. M. L.; Ruijter, E. Org. Lett. 2021, 23, 3100-3104. doi:10.1021/acs.orglett.1c00785

17. Wu, C.-J.; Cao, W.-X.; Chen, B.; Tung, C.-H.; Wu, L.-Z. Org. Lett. 2021, 23, 2135-2139. doi:10.1021/acs.orglett.1c00290

18. Jiang, Z.; Zhou, J.; Zhu, H.; Liu, H.; Zhou, Y. Org. Lett. 2021, 23, 4406-4410. doi:10.1021/acs.orglett.1c01341

19. Aggarwal, T.; Sushmita, S.; Verma, A. K. Org. Biomol. Chem. 2019, 17, 8330-8342. doi:10.1039/c9ob01381d

20. Chaudhari, T. Y.; Tandon, V. Org. Biomol. Chem. 2021, 19, 1926-1939. doi:10.1039/d0ob02274h

21. Sheng, F.-T.; Wang, J.-Y.; Tan, W.; Zhang, Y.-C.; Shi, F. Org. Chem. Front. 2020, 7, 3967-3998. doi:10.1039/d0qo01124j

22. Wang, J.-Y.; Sun, M.; Yu, X.-Y.; Zhang, Y.-C.; Tan, W.; Shi, F. Chin. J. Chem. 2021, 39, 2163-2171. doi:10.1002/cjoc.202100214

23. Sheng, F.-T.; Li, Z.-M.; Zhang, Y.-Z.; Sun, L.-X.; Zhang, Y.-C.; Tan, W.; Shi, F. Chin. J. Chem. 2020, 38, 583-589. doi:10.1002/cjoc.202000022

24. Liu, L.; Zhang, J. L. Chin. J. Org. Chem. 2019, 39, 3308-3309. doi:10.6023/cjoc201900004

25. Tan, B. Chin. J. Org. Chem. 2020, 40, 1404-1405. doi:10.6023/cjoc202000027

26. Mao, Y.; Lu, Y.; Li, T.; Wu, Q.; Tan, W.; Shi, F. Chin. J. Org. Chem. 2020, 40, 3895-3907. doi:10.6023/cjoc202005096

27. Jiang, M.; Zhou, T.; Shi, B. Chin. J. Org. Chem. 2020, 40, 4364-4366. doi:10.6023/cjoc202000083

28. Neto, J. S. S.; Zeni, G. Org. Chem. Front. 2020, 7, 155-210. doi:10.1039/c9qo01315f

29. Tu, M.-S.; Chen, K.-W.; Wu, P.; Zhang, Y.-C.; Liu, X.-Q.; Shi, F. Org. Chem. Front. 2021, 8, 2643-2672. doi:10.1039/d0qo01643h

30. Guan, X.-K.; Zhang, H.; Gao, J.-G.; Sun, D.-Y.; Qin, X.-S.; Jiang, G.-F.; Zhang, G.-L.; Zhang, S. J. Org. Chem. 2019, 84, 12562-12572. doi:10.1021/acs.joc.9b02024

31. Pirovano, V.; Brambilla, E.; Moretti, A.; Rizzato, S.; Abbiati, G.; Nava, D.; Rossi, E. J. Org. Chem. 2020, 85, 3265-3276. doi:10.1021/acs.joc.9b03117

32. Wang, T.; Hoye, T. R. J. Am. Chem. Soc. 2016, 138, 13870-13873. doi:10.1021/jacs.6b09628

33. Sha, F.; Tao, Y.; Tang, C.-Y.; Zhang, F.; Wu, X.-Y. J. Org. Chem. 2015, 80, 8122-8133. doi:10.1021/acs.joc.5b01223
34. Dai, W.; Jiang, X.-L.; Tao, J.-Y.; Shi, F. J. Org. Chem. 2016, 81, 185-192. doi:10.1021/acs.joc.5b02476

35. Chen, S.; Li, Y.; Ni, P.; Huang, H.; Deng, G.-J. Org. Lett. 2016, 18 , 5384-5387. doi:10.1021/acs.orglett.6b02762

36. Ozaki, K.; Zhang, H.; Ito, H.; Lei, A.; Itami, K. Chem. Sci. 2013, 4, 3416-3420. doi:10.1039/c3sc51447a

37. Zheng, X.; Lv, L.; Lu, S.; Wang, W.; Li, Z. Org. Lett. 2014, 16, 5156-5159. doi:10.1021/ol5025053

38. Chen, S.; Li, Y.; Ni, P.; Yang, B.; Huang, H.; Deng, G.-J. J. Org. Chem. 2017, 82, 2935-2942. doi:10.1021/acs.joc.6b02892

39. Lu, C.; Huang, H.; Tuo, X.; Jiang, P.; Zhang, F.; Deng, G.-J. Org. Chem. Front. 2019, 6, 2738-2743. doi:10.1039/c9qo00603f

40. Ni, P.; Tan, J.; Zhao, W.; Huang, H.; Xiao, F.; Deng, G.-J. Org. Lett. 2019, 21, 3687-3691. doi:10.1021/acs.orglett.9b01138

41. Yang, R.-Y.; Sun, J.; Tao, Y.; Sun, Q.; Yan, C.-G. J. Org. Chem. 2017, 82, 13277-13287. doi:10.1021/acs.joc.7b02397

42. Yang, R.-Y.; Sun, J.; Sun, Q.; Yan, C.-G. J. Org. Chem. 2018, 83, 5909-5919. doi:10.1021/acs.joc.8b00196

43. Wang, D.-Q.; Sun, J.; Yan, C.-G. ChemistrySelect 2019, 4, 10550-10554. doi:10.1002/slct.201902407

44. Sun, J.; Yang, R.-Y.; Zhan, S.-C.; Yan, C.-G. ChemistrySelect 2019, 4, 10100-10103. doi:10.1002/slct.201902619

45. Ye, R.; Yan, C.-G. Eur. J. Org. Chem. 2019, 5882-5886. doi:10.1002/ejoc.201900955

46. Zhan, S.-C.; Sun, J.; Liu, R.-Z.; Yan, C.-G. Org. Biomol. Chem. 2020, 18, 163-168. doi:10.1039/c9ob02013f

47. Wang, D.; Sun, J.; Liu, R.-Z.; Wang, Y.; Yan, C.-G. J. Org. Chem. 2021, 86, 5616-5629. doi:10.1021/acs.joc.1c00103

48. Yan, C.; Sun, J.; Yan, C.-G. Chin. Chem. Lett. 2021, 32, 1253-1256. doi:10.1016/j.cclet.2020.08.052

49. Zhan, S.-C.; Fang, R.-J.; Sun, J.; Yan, C.-G. J. Org. Chem. 2021, 86, 8726-8741. doi:10.1021/acs.joc.1c00538

50. Ye, R.; Sun, J.; Han, Y.; Yan, C.-G. New J. Chem. 2021, 45, 5075-5080. doi:10.1039/d0nj06036d

51. Yan, C.; Sun, J.; Han, Y.; Yan, C.-G. J. Org. Chem. 2021, 86, 9263-9279. doi:10.1021/acs.joc.1c00044

52. Zhan, S.-C.; Fang, R.-J.; Sun, J.; Yan, C.-G. Org. Biomol. Chem. 2021, 19, 6322-6327. doi:10.1039/d1ob01113h

53. Zhan, S.-C.; Fang, R.-J.; Yang, R.-Y.; Zhao, R.-F.; Wang, Y.; Sun, J.; Yan, C.-G. New J. Chem. 2021, 45, 15423-15428. doi:10.1039/d1nj02836g 


\section{License and Terms}

This is an Open Access article under the terms of the Creative Commons Attribution License (https://creativecommons.org/licenses/by/4.0). Please note that the reuse, redistribution and reproduction in particular requires that the author(s) and source are credited and that individual graphics may be subject to special legal provisions.

The license is subject to the Beilstein Journal of Organic Chemistry terms and conditions:

(https://www.beilstein-journals.org/bjoc/terms)

The definitive version of this article is the electronic one which can be found at:

$\underline{\text { https://doi.org/10.3762/bjoc.17.159 }}$ 